\title{
Paraffin Oil Pneumonitis During Pregnancy: A Case Report
}

\author{
Moles Athanasios ${ }^{1}$, Fotopoulos Stavros ${ }^{1}$, Cheirakis Emmanouil ${ }^{1}$, Koronaios Vasilios $^{1}$, Kalousis \\ Evangelos $^{2}$, Lintzeri Dimitra ${ }^{2}$, Lazarescu Daria ${ }^{3}$, Grigorakos Leonidas ${ }^{2,3^{*}}$ \\ ${ }^{1}$ REA Maternity Hospital, Athens, Greece \\ ${ }^{2}$ Intensive Care, "KAT” Trauma Hospital of Athens, Kifissia, Athens, Greece \\ ${ }^{3}$ Faculty of Nursing, National and Kapodistrian University of Athens, Greece
}

*Corresponding Author: Leonidas Grigorakos Assoc. Prof., 2 Nikis St, 14561, Kifissia, Athens, Greece, Tel: +30.210.3709522; Fax: +30.210.3709520; E-mail: grigorakos@parliament.gr

Received: 03 April 2018; Accepted: 09 April 2018; Published: 12 April 2018

\begin{abstract}
Acute respiratory distress syndrome (ARDS) is a rare occurrence in pregnant patients and, when it occurs, it usually raises diagnostic dilemmas as it is attributed to many causes. We present the case of a 32-year-old G1P0 woman at 31 weeks of gestation who was hospitalized because of bleeding per vagina and symptoms of gastroesophageal reflux disease (GERD) with episodes of vomiting. Within 72 hours from her admission, she developed dyspnea, cough and hypoxemia and was transferred to our intensive care unit (ICU). She was diagnosed with paraffin oil pneumonitis, treated accordingly and discharged in good health. We highlight medical personnel should keep high suspicion for paraffin oil pneumonitis in pregnant patients with features of GERD who develop ARDS after treatment with liquid paraffin.
\end{abstract}

Keywords: Paraffin oil; Pneumonitis; Acute Respiratory Distress Syndrome; Pregnancy

\author{
Abbreviations \\ GERD $=$ Gastroesophageal Reflux Disease \\ ARDS $=$ Acute Respiratory Distress Syndrome \\ DVT $=$ Deep Venous Thrombosis \\ TEE $=$ Transthoracic Echocardiography \\ $\mathrm{WBC}=$ White Blood Cell
}


$\mathrm{CRP}=\mathrm{C}$-Reactive Protein

HFNO = High Flow Nasal Oxygen

G1P0 $=$ Pregnant for the first time and has not yet delivered

$\mathrm{ALI}=$ Acute Lung Injury

\section{Introduction}

Acute respiratory distress syndrome (ARDS) rarely occurs in the pregnant patients with an estimated incidence of 16-70:100.000 cases [1]. Pregnancy-related ARDS is attributed to many causes and many times poses a diagnostic dilemma. Acute respiratory failure during pregnancy or the peripartum period may be caused by conventional respiratory insult or a pregnancy-specific disorder. The usual differential diagnosis includes pulmonary edema (tocolytics, cardiogenic, preeclampsia-eclampsia), pneumonia, asthma exacerbation, aspiration, pulmonary embolism, amniotic fluid embolism, air embolism, transfusion reactions, pyelonephritis, chorioamnionitis [2].

Routine use of stimulant laxatives is not recommended in pregnancy as there is a risk of lipoid pneumonia occurrence caused by the aspiration of liquid paraffin and mineral/castor oil [3]. The most commonly affected patients are those at the extremes of age who have risk factors for aspiration. The result is either an inflammatory response with regional edema and intralveolar hemorrhage (presenting acutely with cough, fever, and dyspnea) or a 'paraffinoma' with aspirated oils encapsulated in the fibrous tissue (presenting with a mass seen on imaging in an asymptomatic patient) [3].

In this case report, we highlight the difficulty of differentiated diagnosis of acute respiratory failure in pregnancy and the role of paraffin oil administration as a cause of pneumonitis.

\section{Case Presentation}

A 32-year-old G1P0 woman at 31 gestational weeks was hospitalized because of bleeding per vagina and frequent uterine contractions. The patient had 1-2 episodes of vomiting/day for the last 3 days. She also reported frequent heartburn.

Vital signs were normal and the physical examination was unremarkable. She did not report abdominal pain, dyspnea, cough, headache, visual disturbances or chest pain.

Her past medical history was significant for gastroesophageal reflux disease (GERD). Upon admission she did not have ruptured membranes. She was treated with oral nifedipine $20 \mathrm{mg}$ tid, oral progesterone $100 \mathrm{mg}$ tid, oral roxithromycin $300 \mathrm{mg}$ once daily, paraffin oil $20 \mathrm{ml}$ bid, and with betamethasone sodium phosphate intramuscularly. 
Laboratory tests upon admission showed a white blood cell (WBC) count of 12,170/ $\mu$ l and a differential showing $78 \%$ neutrophils. C-reactive protein (CRP) was $0.6 \mathrm{mg} / \mathrm{dl}$ (normal limits $0.0-0.8 \mathrm{mg} / \mathrm{dl}$ ).

On the third day of hospitalization, the patient developed cough and shortness of breath. On physical examination she was dyspneic. Vital signs revealed hypoxia $80 \%$ on room air, tachypnea with a respiratory rate of 34 , a temperature of $36.7^{\circ} \mathrm{C}$, a heart rate of 125 beats per minute (bpm), and blood pressure of 110/60 mm Hg. Her physical exam revealed fine crackles at both lung bases. Heart sounds were normal without murmurs and clicks. The patient was in distress and diaphoretic. There was no facial or hand edema nor tenderness and swelling of the lower extremity. At that time, a chest X-ray showed bilateral infiltrates without bronchograms located in both lower lobes (Figure 1). The electrocardiogram was normal.

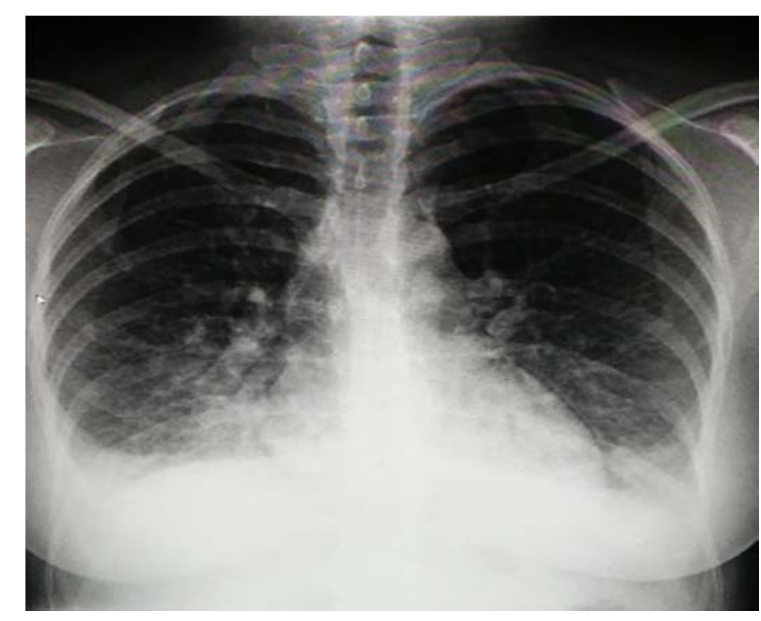

Figure 1: Chest x-ray demonstrating bilateral infiltrates without bronchograms located in both lower lobes

The patient was admitted to the ICU. Broad-spectrum antibiotics, intravenous corticosteroids, and high flow nasal oxygen (HFNO) were administered. Arterial blood gas sampling after initiation of HFNO (air 401/min, $\mathrm{FiO}_{2}: 0.35$ ) showed a $\mathrm{pH} 7.50, \mathrm{PaCO}_{2} 28 \mathrm{mmHg}$, and $\mathrm{PaO}_{2} 73 \mathrm{mmHg}\left(\mathrm{PaO} 2 / \mathrm{FiO}_{2}=208\right)$. The patient was treated with intravenous furosemide without an improvement of her symptoms.

Once arrived in the ICU she was submitted to laboratory tests, which were repeated until release (Table 1).

\begin{tabular}{|l|l|l|l|}
\hline Laboratory parameters & Day 1 & Day 2 & Day 5 \\
\hline WBC & $19,790 / \mu \mathrm{l}$ & $14,440 / \mu \mathrm{l}$ & $10,000 / \mu \mathrm{l}$ \\
\hline CRP $(\mathrm{mg} / \mathrm{dl})$ & 0.01 & 13.73 & 2.8 \\
\hline Platelet count & $198,000 / \mathrm{ml}$ & $213,000 / \mathrm{ml}$ & $235,000 / \mathrm{ml}$ \\
\hline
\end{tabular}

Table 1: Laboratory parameters during ICU stay 
Liver and renal function tests, including prothrombin time, troponin I, and serum albumin level, were within normal limits. There was no proteinuria. A venous duplex Doppler sonography excluded deep venous thrombosis (DVT) while the transthoracic echocardiography (TEE) was normal.

The second day in the ICU the patient was still dyspneic, she had a dry cough, was on HFNO, had a temperature of $37.9^{\circ} \mathrm{C}$, no uterine contractions, normal diuresis, and fine crackles at both lung bases. Her abdomen was soft with normal bowel sounds.

Blood and urine cultures were negative. PCR for influenza was negative. The fetal heart rate was within 130 - 140 bpm rate. Amniocentesis performed that day did not reveal any organisms on Gram stain and culture was negative. The patient was disconnected from the HFNO on the fourth day when she felt much better. She was afebrile, receiving oxygen with nasal canula on $11 \mathrm{t} / \mathrm{min}\left(\mathrm{pH} 7.44, \mathrm{PaCO}_{2} 32 \mathrm{mmHg}\right.$, and $\mathrm{PaO}_{2} 92 \mathrm{mmHg}$ ), still had fine crackles at both lung bases but with improvement.

After five days in the ICU the patient was transferred to the ward. She was hospitalized two more days and then she was discharged home. At the time she was discharged she did not have any dyspnea or cough and the lungs were clear to auscultation.

\section{Discussion}

Respiratory distress in pregnancy is an acute emergency and necessitates rapid assessment and therapy. Approximately $50 \%$ of the cases of pulmonary edema in pregnancy were attributed to tocolytic therapy or cardiac disease. The remaining cases were ascribed to either preeclampsia or iatrogenic volume overload [5]. Our patient received tocolytic therapy with oral nifedipine. Previous studies reveal acute pulmonary failure during tocolysis mainly with beta agonists and intravenous nicardipine [6].

Our patient did not respond to therapy with furosemide and the lung injury persisted for five days. She did not have known cardiac disease and the TEE was normal. There was no elevation in blood pressure and no proteinuria. At the time of acute lung injury (ALI) she was not receiving intravenous fluids, so volume overload was ruled out. She did not have symptoms of infection prior to the development of acute respiratory failure. She was afebrile and the CRP at the time of ALI was normal making the diagnosis of pneumonia unlikely [7].

Intra-amniotic infection was unlikely because the results of amniocentesis were normal and so was the fetal heart rate, while there was no history of asthma and no wheezes. Pulmonary embolism was taken into consideration but a venous duplex Doppler sonography excluded DVT since there was no right ventricle strain, there was no pleuritic pain or hemoptysis [8]. 
More so, amniotic fluid embolism was unlikely because of lack of cardiovascular collapse, seizers or disseminated intravascular coagulation, risk factors such as labor, multiparity, amniotomy, uterine manipulations, trauma [9]. Air embolism was also excluded because there were no intravenous manipulations [10,11].

Aspiration of gastric contents is most common during labor or soon after delivery. This is probably because the effects of sedation, analgesia, increased intraabdominal pressure, and recumbency are added to pre-existing factors that predispose pregnant women to aspiration. Aspiration may induce chemical pneumonitis, airway obstruction, or acute bronchospasm. Features of chemical pneumonitis include an abrupt onset of symptoms and signs, a cough productive of material that resembles gastric contents, diffuse crackles, and chest X-ray infiltrates that are multifocal and predominate in the dependent portions of the lung. In addition, fever is moderate [12]. Lipoid pneumonia is caused by the aspiration of mineral oil when used for constipation and the result is either an inflammatory response with regional edema and intralveolar hemorrhage or a 'paraffinoma' with aspirated oils encapsulated by fibrous tissue. In our case, we considered more possible that the patient's acute respiratory failure was due to pneumonitis from paraffin oil as she was always alert, the aspiration was indolent and the cough was dry, without gastric contents.

To conclude, we highlight that medical personnel should keep high suspicion for paraffin oil pneumonitis in pregnant patients with features of GERD who develop ARDS after treatment with liquid paraffin. Thus, routine use of stimulant laxatives should be avoided during pregnancy.

\section{Competing interests}

The authors declare that there is no conflict of interest regarding the publication of this paper.

\section{References}

1. Rush B, Martinka P, Kilb B, McDermid R, et al. Acute Respiratory Distress Syndrome in Pregnant Women. Obstet Gynecol 129 (2017): 530-535.

2. Catanzarite V, Willms D, Wong D, Landers C, Cousins L, Schrimmer D. Acute respiratory distress syndrome in pregnancy and the puerperium: causes, courses, and outcomes. Obstet Gynecol 97 (2001): 760-764.

3. Powrie R, Greene M, Camann E (eds). De Swiet's Medical Disorders in Obstetric Practice, $5^{\text {th }}$ edition. 2010, Wiley-Blackwell Publications.

4. Betancourt SL, Martinez-Jimerez S, Rossi SE, Truong MT, Carrilo J, Erasmus JJ. Lipoid pneumonia: Spectrum of clinical and radiologic manifestations. AJR Am J Roentgenol 194 (2010): 103.

5. Lapinsky SE. Acute respiratory failure in pregnancy. Obstet Med 8 (2015): 126-132.

6. Kutuk MS, Ozgun MT, Uludag S, Dolanbay M, Yildirim A. Acute pulmonary failure due to pulmonary edema during tocolytic therapy with nifedipine. Arch Gynecol Obstet 288 (2013): 953-954.

7. Graves CR. Pneumonia in pregnancy. Clin Obstet Gynecol 53 (2010): 329-336.

8. Bennett A, Chunilal S. Diagnosis and Management of deep Vein Thrombosis and Pulmonary Embolism in 
Obstet Gynecol Res 2018; 1 (2): 032-037

DOI: 10.26502/ogr006

Pregnancy. Semin Thromb Hemost 42 (2016): 760.

9. Grigorakos L, Markou N, Lazarescu D, Tzortzopoulou K, Gkouni M, Papaioannou E, Bikou M, Moles A. Effective Management of Patients with Amniotic Fluid Embolism in the Intensive Care Unit: Two Case Reports. Int Arch of Med 10 (2017): 527.

10. Bandi VD, Munnur U, Matthay MA. Acute Lung Injury and acute respiratory distress syndrome in pregnancy. Crit Care Clin 20 (2004): 577.

11. Grigorakos L. Evaluating mechanical ventilation in patients with Acute Respiratory Distress Syndrome. Arch Pulmonol Respir Care 4 (2018): 001-005.

12. Son YG, Shin J, Ryu HG. Pneumonitis and pneumonia after aspiration. J Dent Anesth Pain Med 17 (2017): 112.

Citation: Moles Athanasios, Fotopoulos Stavros, Cheirakis Emmanouil, Koronaios Vasilios, Kalousis Evangelos, Lintzeri Dimitra, Lazarescu Daria, Grigorakos Leonidas. Paraffin Oil Pneumonitis During Pregnancy: A Case Report. Obstetrics and Gynecology Research 1 (2018): 032-037.

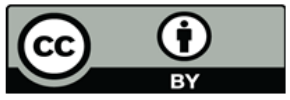

This article is an open access article distributed under the terms and conditions of the $\underline{\text { Creative Commons Attribution (CC-BY) license } 4.0}$ 